\title{
Scanning laser fundus perimetry before laser photocoagulation of well defined choroidal neovascularisation
}

Klaus Rohrschneider, Roland Glück, Matthias Becker, Frank G Holz, Friedrich E Kruse, Thomas Fendrich, Hans E Völcker

\begin{abstract}
Aim-To assess the centre of fixation before laser photocoagulation of well defined juxtafoveal or extrafoveal choroidal neovascularisation (CNV) secondary to age related macular degeneration (AMD), and to better predict visual function after treatment using scanning laser ophthalmoscope (SLO) fundus perimetry.

Methods-19 consecutive eyes with juxtafoveal or extrafoveal CNV were examined by fundus perimetry before and after laser treatment with documentation of the fixation point using the SLO. The stability of fixation was defined as standard deviation around the mean fixation point. Overlays of fluorescein angiographic pictures and fundus perimetry were obtained using image analysis software.

Results-Fundus perimetry allowed accurate determination of the centre of fixation. Overlays demonstrated the precise geographic relation of the angiographically detectable foveal margin of the CNV and the centre of fixation. Thereby, prediction of the visual outcome with regard to reading ability was facilitated. Stability of fixation did not change significantly after treatment.

Conclusions-Fundus perimetry using the SLO was helpful in patients who underwent laser treatment for juxtafoveal or extrafoveal CNV secondary to AMD and may aid the pretreatment counselling of such patients.

(Br f Ophthalmol 1997;81:568-573)
\end{abstract}

Ophthalmology

University of

Heidelberg,

Heidelberg, Germany

K Rohrschneider

R Glück

M Becker

F G Holz

F E Kruse

H E Völcker

Institute of Applied Physics, University of Heidelberg,

Heidelberg, Germany

T Fendrich

Correspondence to: Dr Klaus Rohrschneider, Univ-Augenklinik, Im Neuenheimer Feld 400, D-69120 Heidelberg, Germany.

Accepted for publication 6 March 1997

Various studies including the Macular Photocoagulation Study have demonstrated a beneficial effect of laser photocoagulation in presence of juxtafoveal and extrafoveal well defined 'classic' choroidal neovascularisation (CNV) secondary to age related macular degeneration. ${ }^{12}$ Besides anatomic successthat is, complete destruction of the choroidal neovascular net by laser treatment, functional outcome with regard to central visual acuity and the ability to read are important to the patient. In order to preserve the patient's visual function several measures are of interest. Besides central visual acuity, which is tested routinely in clinical practice, the central visual field, the actual location of the centre of fixation, as well as the stability of fixation represent important additional information. How- ever, no tests for the determination of fixation and its stability have been introduced into routine clinical work to date.

Conventional perimetry, either performed as static computerised perimetry or kinetic perimetry using the Goldmann perimeter, often lacks reliable results with respect to usually relatively small paracentral visual field defects. In addition, movement of the centre of fixation leads to concurrent changes of the location of scotomas. Therefore, documentation of the centre of fixation is often unsatisfactory using these techniques.

The advent of scanning laser systems with several independent lasers has given us the opportunity simultaneously to visualise the fundus and perform various functional tests, ${ }^{3-5}$ including the documentation of the exact point of fixation at the fundus. ${ }^{6}$

The purpose of this study was to perform fundus perimetry using the scanning laser ophthalmoscope (SLO) with a newly developed static threshold fundus perimetry ${ }^{7}$ to document the area of fixation before and after laser treatment of juxtafoveolar and extrafoveolar $\mathrm{CNV}$ and to assess for the possibility of more accurate prediction of the visual outcome. In addition, we evaluated whether the knowledge of these functional findings may provide valuable information in addition to the morphological and angiographic appearance. We therefore compared patients with and without foveal or central fixation in the presence of neovascular membranes.

\section{Patients and methods}

PATIENTS

Nineteen consecutive eyes of 19 patients (five men and 14 women aged 52 to 83 years (70 (SD 8.5) years)) with juxtafoveolar and extrafoveolar well defined 'classic' choroidal neovascularisation based on the fluorescein angiographic findings were enrolled in our study and were followed after laser treatment. Routine ophthalmic examination included visual acuity, and stereoscopic fundus examination using the $78 \mathrm{D}$ lens or the Goldmann three mirror lens. Fluorescence angiography was performed fluorescein dye.

Reading ability was evaluated by asking the patients about their subjective assessment of visual performance and reading ability. using the SLO after injection of $10 \mathrm{ml} \mathrm{5 \%}$ 
SCANNING LASER OPHTHALMOSCOPE

The principle of the SLO (Rodenstock, Munich, Germany) has been described earlier. ${ }^{89}$ In brief, the SLO projects a helium neon $(\mathrm{HeNe})$ laser beam $(632.8 \mathrm{~nm})$ and an infrared diode laser $(780 \mathrm{~nm})$ simultaneously onto the fundus with an image size of 33 by 21 degrees. The amount of light projected onto the retina is well below the limit set by the ANSI for
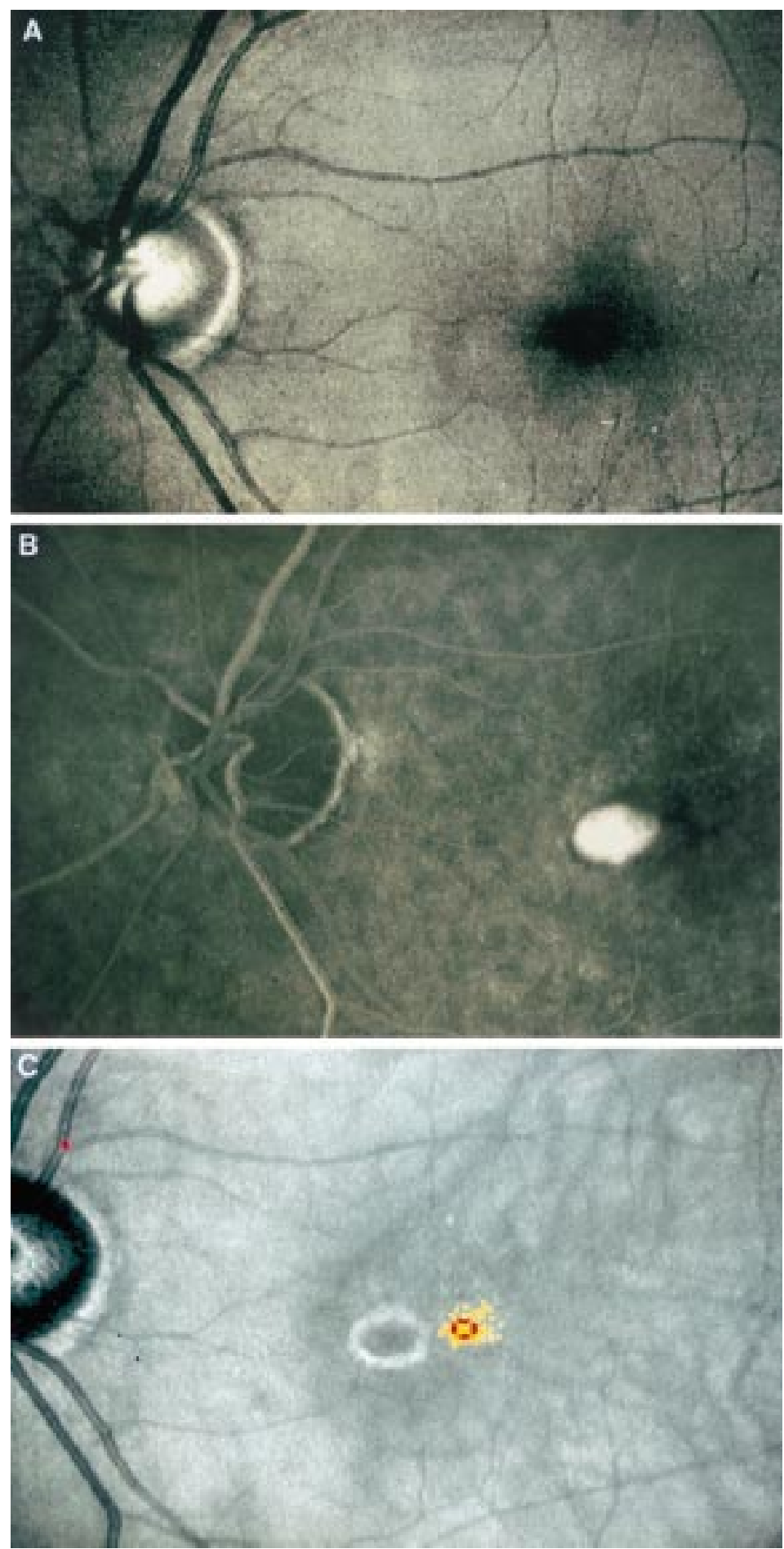

Figure 1 Comparison of the visibility of the choroidal neovascularisation (CNV) using different illumination wavelengths in a left eye. (A) Fundus image using the SLO with argon blue light, as obtained during beginning of the angiography, does not show the CNV. (B) Midphase of angiography clearly outlines the choroidal neovascular membrane. (C) Fundus image obtained using infrared diode laser of the SLO with mean fixation point and a circle demonstrating the standard deviation of fixation; the CNV is clearly visible. lasers. ${ }^{10}$ The HeNe laser used for generation of the background and stimulus illumination is modulated via an acousto-optic modulator. ${ }^{9}$ The image of the retina is acquired simultaneously by illumination with infrared laser light through a set of nearly confocal apertures. ${ }^{11}$

FUNDUS EXAMINATION

Static threshold fundus perimetry was performed using the SLO within 48 hours before and within 3-5 weeks after laser treatment after informed consent was obtained. In addition, a subset of nine patients was followed for up to 180 days, including those with persistence or recurrence of the CNV. We used newly developed software with the help of an additional personal computer as described earlier. ${ }^{7}$ According to further development of the software the stimulus can be projected exactly onto the predefined position by the help of a landmark setting on the real time image. ${ }^{12}$ During each stimulus presentation the fundus image is digitised and a correction for small movements following the initial landmark definition is performed. Background illumination was set to $10 \mathrm{~cd} / \mathrm{m}^{2}$. The fixation target was a cross measuring $1.5 \times 1.5$ degrees with a central opening of 0.5 degrees which allowed us to project a stimulus into the centre of fixation. Stimulus presentation time was 120 ms. Stimulus intensity could be varied in 0.1 $\log$ steps from 0 to $21 \mathrm{~dB}$, where $0 \mathrm{~dB}$ represented the brightest luminance of 71 $\mathrm{cd} / \mathrm{cm}^{2}$. In addition, stimulus intensities of 23.6 and $26 \mathrm{~dB}$ could be projected. Stimulus diameter amounted to $0.38 \times 0.41$ degrees $(6 \times 10$ pixel) horizontally and vertically (comparable to Goldmann III).

In addition to static threshold fundus perimetry we performed kinetic perimetry in eyes with absolute or very deep scotomas in order to obtain a more precise definition of the size of the scotomas. Therefore, stimulus size was changed to the equivalent of Goldmann II while the intensity was variable to the same degree, as mentioned above. ${ }^{13}$

The point of fixation was determined during either perimetric procedure for each stimulus presentation by the help of the landmark setting. From the single fixation points of the static perimetry the computer calculated the mean fixation point (MFP) as the centre of gravity. ${ }^{6}$ The number of stimulus presentations varied between 200 and 496 (344 (SD 83)). Furthermore, we calculated the deviation around this MFP for an appropriate determination of the stability of fixation. The variation of the determination of this single point is less than 0.5 degrees; for the MFP the acuity is better than 0.2 degrees.

In some eyes with poor stability of fixation we performed another fixation task, in addition to the previous examination, whereby the patient was asked to fixate the same projected target as stable as possible and to determine the moment of best fixation by pressing a button. This procedure was repeated 40 times which enabled us more precisely to document the subjective and more stable location of fixation. 

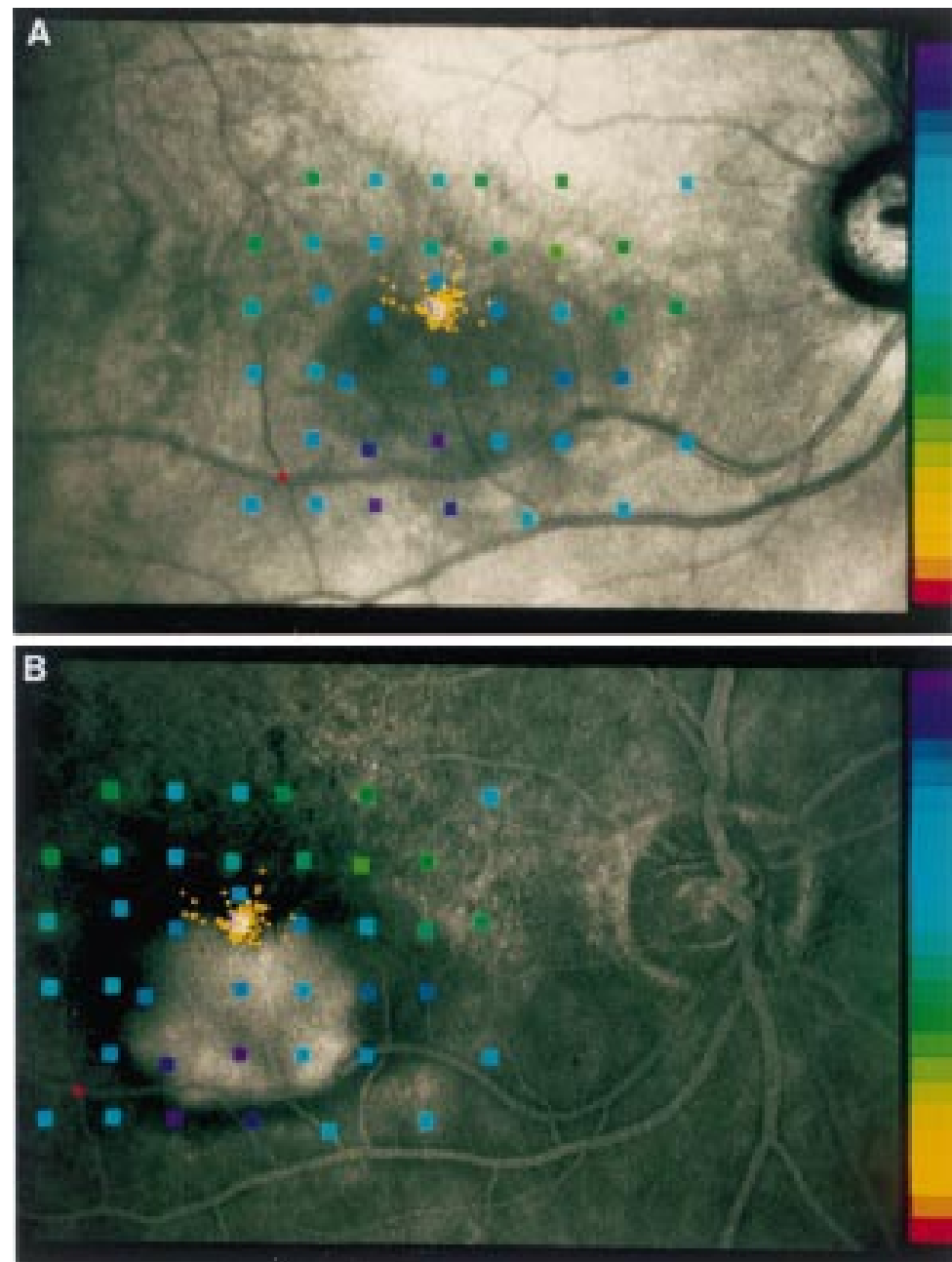

Figure 2 Results of static fundus perimetry in a right eye of a patient with choroidal neovascularisation $(C N V)$. (A) Standard image with threshold values according to the right hand scale with brightest stimuli at the top and smallest contrast between stimuli and background at the bottom. Single fixation points are drawn as yellow dots. (B) Results converted onto the mid time sequence of fluorescence angiography which enables direct visualisation of mean fixation point in relation to classic CNV.

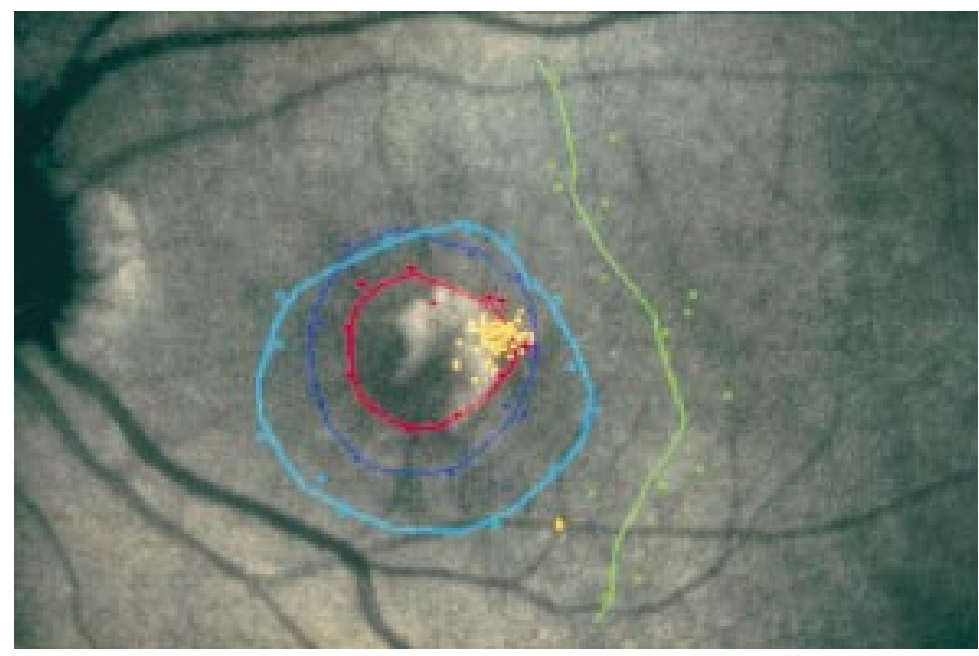

Figure 3 Example of subjectively poor visual outcome in a left eye after treatment although visual acuity by conventional testing has remained unchanged. The mean fixation point is located at the right margin of the laser scar at the fundus (yellow points). This will lead to a scotoma at the left of the point of fixation which makes reading more difficult owing to missing of the words or digits which have just been read. Kinetic perimetry shows an absolute scotoma ( $0 \mathrm{~dB}$, Goldmann II, red circle) and a larger relative scotoma (5 dB purple, $10 \mathrm{~dB}$ blue, $15 \mathrm{~dB}$ green).
LASER TREATMENT

Laser treatment was performed according to the indication and technique reported in the MPS studies by the use of an argon green laser. ${ }^{12}$ Before treatment signed informed consent was obtained. The time interval between angiography and treatment did not exceed 24 hours. ${ }^{14}$

\section{Results}

Determination of the mean fixation point (MFP) was achieved during fundus perimetry in all patients. Owing to the infrared light used to obtain the image the size and location of the CNV was sometimes more visible during SLO fundus perimetry than in the conventional fundus image or in the initial red-free photograph of the fluorescein angiography (Fig 1). ${ }^{15}$ In addition, the results of the fundus perimetry could be directly transposed onto the images obtained during angiography in those eyes in which both examinations were performed using the SLO (Fig 2). This allowed us to locate precisely the centre of fixation in relation to the foveal border of the neovascular membrane, which facilitated laser treatment.

The location of the mean fixation point at the fundus image in relation to the margin of the CNV in all patients is shown in Table 1. Subjective visual outcome tended to be better if the MFP was located on the left (that is, temporal in a right eye) or superior margin of the choroidal membrane even when visual acuity showed no improvement. Ten of 12 patients with such location noted stable or improved visual performance. In contrast, four of seven patients with location of the MFP at the right or inferior margin of the $\mathrm{CNV}$ reported subjective worsening even if the visual acuity remained the same (Fig 3, Table 1).

In eight eyes the classic $\mathrm{CNV}$ was located juxtafoveolar with a location of the mean fixation point just at the border of the membrane. All of these eyes were treated with entire coverage of the neovascular net. However, an attempt was made not to destroy the MFP. Reading ability improved in these eyes owing to a decrease in metamorphopsia and an increase of stability of fixation. However, in two of these eyes there was a recurrence of the $\mathrm{CNV}$ with subfoveolar extension during the subsequent follow up which was associated with marked deterioration in visual acuity.

The scotomas overlying the neovascular net were absolute if the size of the CNV was greater than $1 \mathrm{~mm}^{2}$ in all eyes. We could not find a significant relation between the size, the deepness of the scotomas, and the initial visual acuity. Nevertheless, in the presence of an absolute scotoma of the same size before and after laser treatment patients noted an initial improvement of the visual function with regard to the decrease of metamorphopsia.

While eight eyes initially showed a central fixation with a median visual acuity of 0.4 (0.16 to 0.5$)$, in 11 eyes the mean fixation point had moved away from the fovea and visual acuity ranged from $1 / 15$ to 0.5 (median 0.3 ). We observed a movement of the MFP towards the fovea after laser treatment in six of 


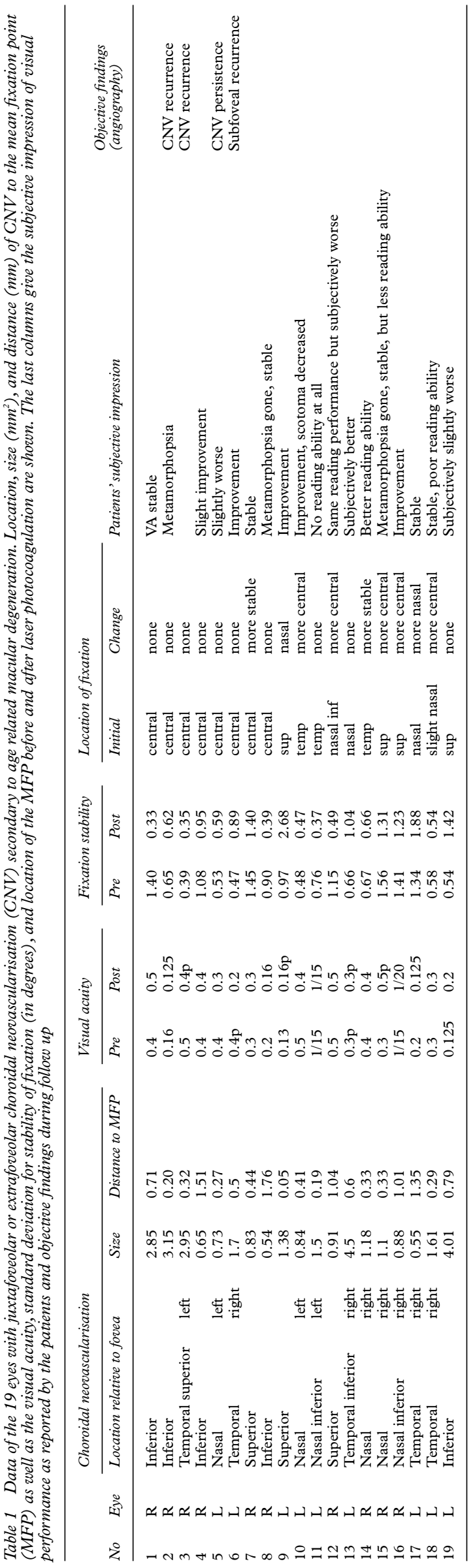

the latter eyes while only one showed a further movement more nasally. In conjunction with these changes, the visual performance - that is, the reading ability and subjective impression, showed improvement or stability in all but one of these 11 eyes (Table 1 ).

Regarding the mean stability of fixation we did not observe a significant change after treatment compared with the initial values (Table 1). Out of five eyes with extrafoveal fixation which showed a slight movement of the MFP towards the fovea after treatment, only one showed an improvement in central visual acuity. Three of these eyes had better stability of fixation.

\section{Discussion}

Visual rehabilitation is the major aim of patients suffering from AMD. Laser treatment of classic choroidal neovascularisation has been shown to be beneficial to the patients when compared with non-treatment, especially if the CNV is located extrafoveolar or juxtafoveolar. ${ }^{1617}$ Regarding the MPS guidelines, the precise localisation of the centre of the foveal avascular zone is an anatomical concept not a functional one with regard to the point of fixation. Although these appear to correspond, our findings show that there may be a movement of the point of fixation away from the fovea. Nevertheless, the aim of laser treatment is preservation of function and, thus, to correlate morphological appearance with functional characteristics. However, determination of the centre of fixation may be difficult in eyes with CNV especially when the fundus morphology changed as a result of the underlying disease process. In the past we applied simple techniques to determine the centre of fixation during laser treatment-for example, by projecting the aiming beam onto the fundus and asking the patient to fixate the laser spot. While some patients will be able to fixate the beam and therefore allow safe treatment many others are not able to fixate stably enough for a variety of reasons. In these patients the use of conventional techniques for the determination of the centre of fixation is of limited value. Standard fundus photography with projection of a fixation target needs an high illumination which makes fixation even more difficult. ${ }^{18}$ In contrast, the scanning laser ophthalmoscope allows us simultaneously to visualise the fundus during projection of many different targets or stimuli under more physiological circumstances. Owing to the use of an infrared laser the level of background illumination needed is only $1-10 \mathrm{~cd} / \mathrm{m}^{2}$. This allows documentation of the point of fixation at the fundus either during fundus perimetry or as an isolated task. The option of a simultaneous image of the fundus enables us to document the point of fixation with an area indicating the stability of fixation calculated as the standard deviation around the mean fixation point. ${ }^{6}$ Since this measurement is calculated from single digitised images obtained during perimetry there is only minimal difference of the mean fixation point between different observers (<0.3 degrees, unpublished data). The varia- 

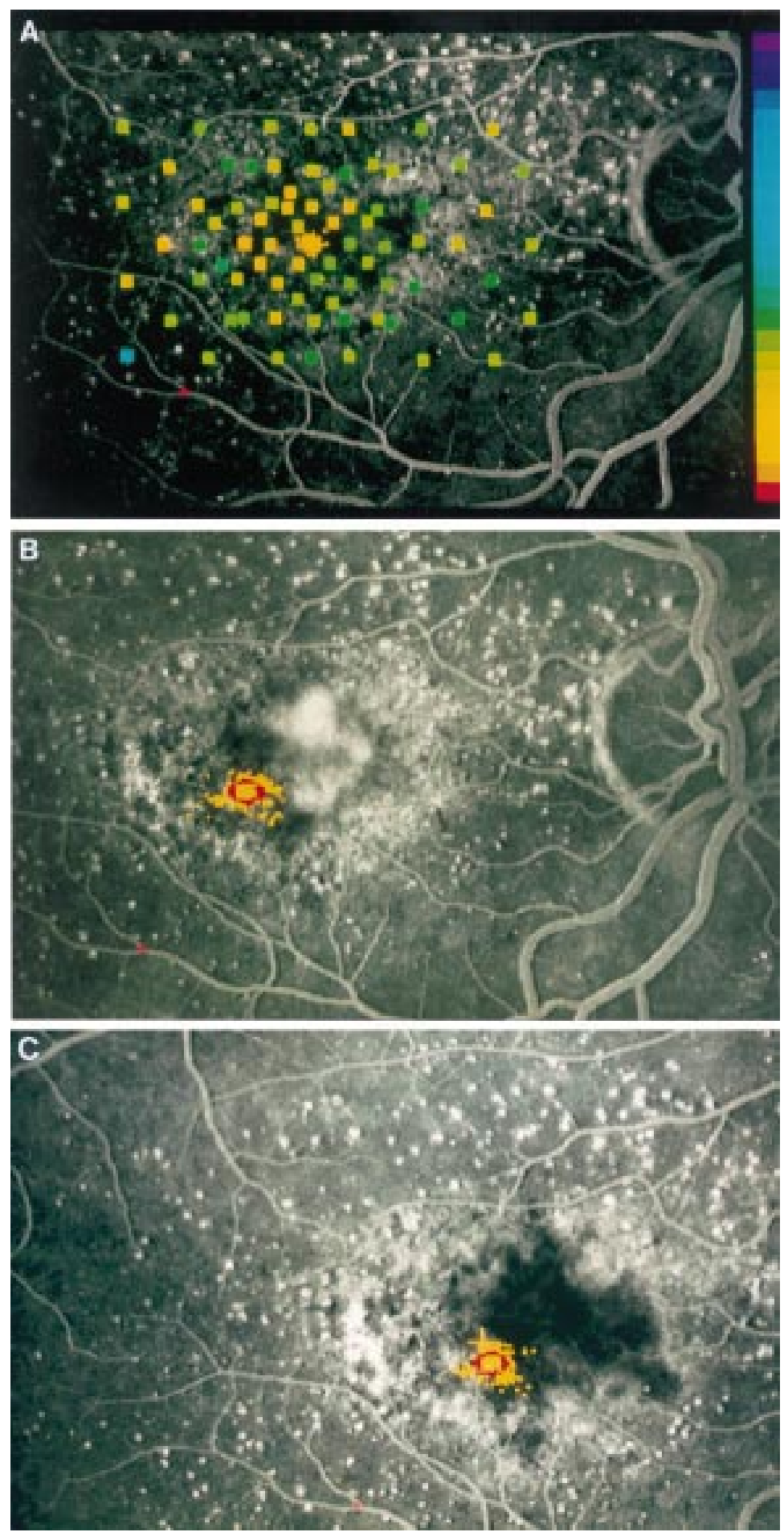

Figure 4 Right eye of a 67-year-old woman first presenting with decrease of visual acuity in the left eye. Right eye showed nearly normal perimetric results with the SLO (right hand scale with brightest stimuli at the top and smallest contrast between stimuli and background at the bottom) and central fixation (yellow dots) while angiography demonstrates macular degeneration without defined leakage (A). (B) Six months later juxtafoveolar CNV nasally of the fovea can be seen during fluorescein angiography, mean fixation point has shifted temporally and stability of fixation is worse (red circle). (C) Four months after laser treatment no recurrence can be seen, visual acuity is 0.4 , and reading ability is improved subjectively. Location and stability of fixation as measured during SLO perimetry are about the same. these examinations are obtained in an identical format. This allows projection of the results of fundus examination onto the angiographic images and, therefore, more accurate knowledge of the distance between preferred fixation and foveal margin of the CNV (Fig 2). Using this composite image, laser treatment may be more accurate and safer. In addition, digital image processing systems may allow image processing in a similar fashion even when different image sources are used.

Moreover, in eyes with juxtafoveolar membranes it sometimes may not be readily possible to assess whether or not the CNV is juxtafoveal or just subfoveolar according to MPS criteria especially when macular morphology is abnormal; fundus visualisation is blurred due to media opacities; and as a result the central avascular zone is not clearly demarcated. In those eyes the exact knowledge of the fixation may play a role in the decision regarding laser photocoagulation even when the preferred fixation point is not at the fovea.

The prediction of the visual outcome may be helpful in the management of patients suffering from exudative AMD. Counselling of these patients regarding the natural course is important, especially when the first eye has already lost central vision, as well as discussion of benefit and potential risk resulting from therapy. According to the results of the MPS Group laser treatment results in a better visual acuity compared with non-treatment after 5 years. ${ }^{19}$ Nevertheless, up to $40 \%$ of the patients suffering from choroidal neovascularisation of age related origin will not benefit from treatment.

Our results indicate that the location of the CNV in relation to the MFP perhaps may predict subjective improvement or worsening after therapy. Location of the CNV inferiorly or at the right of the mean fixation point at the fundus - that is, nasally in a right eye or temporally in a left eye, was associated with a tendency of more subjective improvement of reading ability after laser treatment. It is known that in patients suffering from central scotoma due to $A M D$, as well as juvenile macular dystrophy, the point of fixation shifts towards comparable locations - that is, to the left or superior of the central scotoma at the fundus. ${ }^{20}$ Therefore, it may be assumed that scotomas on the left are more annoying even without treatment.

Besides the location of the choroidal neovascularisation the functional impairment before the treatment is of interest. As our results indicate patients with an absolute scotoma overlying the CNV will not notice much change of their visual function after laser photocoagulation especially when the scotoma does not increase in size. On the other hand these patients often primarily suffer from macular oedema or accumulation of subneurosensorial fluid which may lead to movements of the point of fixation away from the fovea even before treatment (Fig 4). Decrease of the oedema after treatment may lead to a reduction in metamorphopsia which causes a subjective improvement, although we could demonstrate an increase in central visual acuity
SLO can be used for fundus examination also for fluorescence or indocyanine green angiography, the resultant images from all

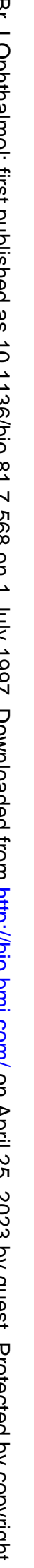


in only one case. When comparing patients with central fixation with those in whom the preferred fixation has moved before laser photocoagulation we found that the latter group showed a tendency to fixate more centrally after treatment and the majority of these eyes had a stable or improved visual outcome $(10 / 11)$. On the other hand in eyes with central fixation the laser scar may involve the fovea following treatment. This results in decreased visual acuity and instable fixation.

In conclusion, fundus perimetry with the option of detection of the fixation point as well as the stability of fixation would not influence the indication for laser treatment according to the criteria reported in several randomised trials. ${ }^{1219}$ However, further studies and a longer follow up are needed to evaluate the clinical value of the knowledge of the area of fixation before and after laser treatment. This new tool may facilitate evaluation of the accurate treatment, and a more precise forecast of the visual outcome.

Supported in part by the Deutsche Forschungsgemeinschaf (DFG) Vo 437/2-1. The authors have no proprietary interest in any of the materials used in this study.

1 Macular Photocoagulation Study Group. Argon laser photocoagulation for senile macular degeneration: results of a randomized clinical trial. Arch Ophthalmol 1982;100:912-8.

2 Moorfields Macular Study Group. Treatment of senile macular degeneration: a single-blind randomised trial by argon laser photocoagulation. Br f Ophthalmol 1982;66 745-53.

3 Van de Velde FJ, Jalkh AE, Katsumi O, Hirose T, Timberlake GT, Schepens CL. Clinical scanning laser ophthalmoscope applications: an overview. In: Nasemann JE, Burk ROW, ed. Scanning laser ophthalmoscopy and tomography. Munich: Quintessenz, 1990: 35-47.

4 Sjaarda RN, Frank DA, Glaser BM, Thompson JT, Murphy RP. Resolution of an absolute scotoma and improvement of relative scotoma after successful macular hole surgery. $\mathrm{Am}$ r Ophthalmol 1993;116:129-39.
5 Sunness JS, Bressler NM, Maguire MG. Scanning laser ophthalmoscopic appearance of the pattern of visual loss in age-related geographic atrophy of the macula. $A m f$ Ophthalmol 1995;119:143-51.

6 Rohrschneider K, Becker M, Kruse FE, Fendrich T, Völcker HE. Stability of fixation-results of funduscontrolled examination using the scanning laser ophthalmoscope. German f Ophthalmol 1995;4:197-202.

7 Rohrschneider K, Fendrich T, Becker M, Krastel H, Kruse FE, Völcker HE. Static fundus perimetry using the scanning laser ophthalmoscope with an automated threshold strategy. Graefes Arch Clin Exp Ophthalmol 1995;233: $743-9$.

8 Webb RH, Hughes GW, Delori FC. Confocal scanning laser ophthalmoscope. Appl Optics 1987;26:1492-9.

9 Plesch A, Klingbeil U. Optical characteristics of a scanning Plesch A, Klingbeil U. Optical characteristics of
laser ophthalmoscope. SPIE 1989;1161:390-8.

10 Laser Institute of America. American national standard for the safe use of lasers. Toledo, OH: The Institute, 1986 (ANSI Z 136.1.1986).

11 Van de Velde FJ, Jalkh AE, Elsner AE. Microperimetry with the scanning laser ophthalmoscope. In: Mills RP, Heijl A, ed. Perimetry update, 1990/91. Amsterdam: Kugler, 1991: 93-101.

12 Sunness JS, Schuchard RA, Shan N, Rubin GS, Dagnelie G, Haselwood DM. Landmark-driven fundus perimetry using the scanning laser ophthalmoscope. Invest Ophthalmol Vis Sci 1995;36:1863-74.

13 Rohrschneider K, Becker M, Fendrich T, Völcker HE. Kinetische funduskontrollierte Perimetrie mit dem Scanning-Laser-Ophthalmoskop. Klin Monatsbl Augenheilkd 1995;207:102-10.

14 Klein ML, Jorizzo PA, Watzke RC. Growth features of choroidal neovascular membranes in age-related macular degeneration. Ophthalmology 1989;96:1416-21.

15 Delori FC, Gragoudas ES, Francisco R, Pruett RC. Monochromatic ophthalmoscopy and fundus photography. Arch Ophthalmol 1977;95:861-8.

16 Macular Photocoagulation Study Group. Recurrent choroidal neovascularization after argon laser photocoagulation for neovascular maculopathy. Arch Ophthalmol 1986;104: 503-12.

17 Macular Photocoagulation Study Group. Laser photocoagulation of subfoveal neovascular lesions in age-related macular degeneration. Results of a randomized clinical trial. Arch Ophthalmol 1991;109:1220-31.

18 Von Noorden GK, Allen L, Burian HM. A photographic method for the determination of the behavior of fixation. Am f Ophthalmol 1959;48:511-4.

19 Macular Photocoagulation Study Group. Argon laser photocoagulation for neovascular maculopathy. Five-year
results from randomized clinical trials. Arch Ophthalmol 1991;109:1109-14.

20 Aulhorn E, Michelfelder F. Vergleich der Fixationsverlagerung bei organischen Maculaerkrankungen und beim physiologischen Dunkelskotom. Ber Dtsch Ophthalmol Ges 1972;71:487-91. 